\title{
Lumbar punctures: use and diagnostic efficiency in emergency medical departments
}

\author{
Bilal Majed • Hélène Zephir • \\ Valérie Pichonnier-Cassagne - Yazdan Yazdanpanah • \\ Philippe Lestavel • Pierre Valette • Patrick Vermersch
}

Received: 9 July 2009 /Accepted: 30 August 2009 /Published online: 19 November 2009

(C) Springer-Verlag London Ltd 2009

\begin{abstract}
Background Lumbar punctures (LP) are regularly and effortlessly used in the emergency medical departments (EMD). LP use and efficiency have not been fully explored in the published literature.

Aims The goal of this study was to assess in a French EMD, the frequency of use and the diagnostic efficiency of LP, the final diagnoses, and related medical practices.

Methods We retrospectively studied all patients who underwent an LP after admission to our adult EMD in 2004 and 2005. Patients' medical files were reviewed to collect
\end{abstract}

The views expressed in this paper are those of the author(s) and not those of the editors, editorial board or publisher.

\footnotetext{
B. Majed $(\bowtie) \cdot$ V. Pichonnier-Cassagne $\cdot$ P. Valette

Emergency Medicine Department,

Unit of Epidemiology \& Clinical Research,

SAMU 62/SMUR/SU/USC, Arras General Hospital,

Boulevard Besnier,

62000 Arras, France

e-mail: bmajed@free.fr

B. Majed

Biostatistics Department, Curie Institute,

26 rue d'Ulm,

75005 Paris, France

H. Zephir $\cdot$ P. Vermersch

Neurology Department, Roger Salengro Hospital, CHRU de Lille, 59000 Lille, France

Y. Yazdanpanah

Service des Maladies Infectieuses et du Voyageur,

Centre Hospitalier de Tourcoing,

135 rue du Président Coty, B.P.619, 59208 Tourcoing, France

P. Lestavel

Emergency Department, Hénin-Beaumont Polyclinic,

Route de Courrière,

62256 Hénin-Beaumont, France
}

clinical and paraclinical features. We defined efficient LP as abnormal LP, which confirmed a suspected diagnosis in an emergency setting.

Results A total of 247 patients, representing $0.5 \%$ of all admissions, underwent an LP. LP were efficient in fewer than $15 \%$ of cases and confirmed aseptic meningitis (8.5\%), bacterial meningitis (2.4\%), Guillain-Barré syndromes (1.6\%), subarachnoid hemorrhages (SAH, 0.4\%), and carcinomatous meningitis $(0.4 \%)$. The principal differential diagnoses were infections outside the central nervous system (CNS, 30\%), noninfectious neurological disorders (28.7\%), and benign headaches (14.2\%). The main assumed LP indications were to search for CNS infection $(62 \%)$ and for SAH (25\%). LP efficiency decreased dramatically according to patients' age. Brain imaging was performed in $90 \%$ of patients prior to LP. Excessive use of polymerase chain reaction to detect herpes simplex in cerebrospinal fluid was observed. The only LP complications found were postdural puncture headaches ( $6.1 \%$ of cases). The rate of traumatic LP was $17 \%$.

Conclusions Our results are in accordance with the few published surveys on this topic. LP efficiency is modest but must be considered in light of the seriousness of suspected diagnoses. However, the search for differential diagnoses should not be neglected.

Keywords Lumbar puncture - Efficiency - Emergency medical department $\cdot$ Meningitis $\cdot$ Diagnosis $\cdot$ Medical practice assessment
Abbreviations
CNS Central nervous system
CSF Cerebrospinal fluid
EMD Emergency medical department
GBS Guillain-Barré syndrome 
LP Lumbar puncture(s)

PCR Polymerase chain reaction

PDPH Postdural puncture headaches

SAH Subarachnoid hemorrhage

\section{Introduction}

Lumbar punctures (LP) are regularly and effortlessly used in emergency medical departments (EMD) [1]. They are essentially employed [2] in order to confirm a central nervous system (CNS) infection [3] or a subarachnoid hemorrhage (SAH) [4], whilst brain imaging is not contributive. LP can also be useful when an acute inflammatory polyradiculoneuropathy (Guillain-Barré syndrome, GBS) is suspected [5]. These disorders can compromise patients' vital and functional prognoses [3, 6, 7]. Therefore, efficient diagnosis strategies (using LP) are required for EMD patients with suspected meningitis, SAH or GBS.

However, LP use and efficiency have not been fully explored in the published literature. Considering published literature data, only few surveys studied the use and the diagnostic efficiency of LP in the EMD $[1,5,8,9]$. Furthermore, none of the published studies used French populations.

LP efficiency (yield) is assumed to be modest especially among elderly patients [10] when an infection of the CNS is suspected. Some findings [1] would appear to suggest that the examination of cerebrospinal fluids (CSF) in the EMD may not always be necessary and that some CSF tests may be excessive. Considering SAH and GBS, while these diseases are infrequently supported by LP results in the EMD [5, 9], missed diagnoses of SAH in the EMD have been reported [11]. Hence, there is a need for further investigations to explore the use and the efficiency of LP performed in the EMD. The main associated investigations, such as computed tomography (CT) of the brain prior to LP [3, 12-17] and CSF tests [18-20], are also important to assess.

In view of the lack of published literature on the use of LP in the EMD, we conducted an observational retrospective study to investigate the frequency of LP use in an emergency setting, the diagnostic efficiency of LP, and the usefulness of CSF tests. We also assessed medical practices associated with the use of LP, such as performing a CT scan prior to an LP; LP complications were also investigated.

\section{Material and methods}

Population studied

We performed a retrospective study, selecting all patients who underwent an LP in the adults' EMD of a general hospital in Arras, in the north of France, between January 2004 and December 2005. The patients studied were those who underwent an LP during the phase of care in the EMD. The CSF results relating to the first successful LP procedure for each admission were collected as well as patients' clinical, biological, and brain imaging findings.

Methods

We assessed the frequency of LP use in our EMD compared to the number of admissions (in 2004 and 2005). We reviewed the medical files of the selected patients. Final diagnoses, patients' clinical features, CSF test findings, and the other paraclinical explorations (CT) were assessed and entered in a database. The retrospective design of our survey led us to assess LP indications and final diagnoses using independent examinations of patients' medical files performed by two emergency physicians. Dates of admission, brain $\mathrm{CT}$, and LP were also available.

We conducted a descriptive analysis to explore patients' features and to assess associations with final diagnoses. Efficient LP were defined as abnormal LP, which confirmed suspected specific diagnoses in an emergency setting (infections of the CNS, SAH, and GBS).

Proportions were computed with confidence intervals of 95\% (95\% CI) and chi-square tests were used [21]. Standard CSF test findings were compared according to the type of meningitis diagnosed: aseptic (lymphocytic) or bacterial. In view of the expected modest efficiency of LP in the EMD, nonparametric tests were used where necessary. S-Plus statistical software was used for the data analysis.

\section{Results}

Frequency of LP use

A total of 247 patients underwent an LP after admission to the EMD between January 2004 and December 2005. There were 46,483 admissions during this period. Thus, $0.53 \%$ (0.47-0.60) of admitted patients underwent an LP. When analyzed monthly, the proportion of patients who underwent an LP varied widely, from a minimum of $0.24 \%$ to a maximum of $0.94 \%$. Nonetheless, no significant difference was found that could indicate a possible seasonal variation in the use of LP $(p=0.28)$.

Patients' clinical features

In our population, the median age was 52 years (36-72), and the sex ratio (male to female) was 1.12. 
Table 1 Distribution of medical records, clinical symptoms found, assumed LP indications, the performance of CT before LP, and final diagnoses in the presence of efficient LP according to patients' age (coded in three classes). The frequency and the proportion (\% in parentheses) of patients' features are indicated among each subpopulation, defined according to age. Significant distribution differences according to patients' age are assessed using chi-square tests

\begin{tabular}{|c|c|c|c|c|c|}
\hline & \multicolumn{3}{|l|}{ Age (years) } & \multirow[t]{2}{*}{ Total } & \multirow[t]{2}{*}{$p$} \\
\hline & Less than 35 & Between $35 \& 65$ & Over 65 & & \\
\hline$n(\%)$ & $56(22.7)$ & $104(42.1)$ & $87(35.2)$ & $247(100)$ & - \\
\hline \multicolumn{6}{|l|}{ Medical records } \\
\hline Neurological records & $1(1.8)$ & $27(26)$ & $36(41.4)$ & $64(25.9)$ & $<0.001$ \\
\hline Alcoholism & $2(3.6)$ & $26(25)$ & $8(9.2)$ & $36(14.6)$ & $<0.001$ \\
\hline Diabetes & $0(0)$ & $7(6.7)$ & $15(17.2)$ & $22(8.9)$ & - \\
\hline Headaches & $7(12.5)$ & $11(10.6)$ & $1(1.1)$ & $19(7.7)$ & - \\
\hline \multicolumn{6}{|l|}{ Clinical symptoms } \\
\hline Nonfocal neurological symptoms (confusion) & $15(26.8)$ & $47(45.2)$ & $55(63.2)$ & $117(47.4)$ & $<0.001$ \\
\hline Fever & 11(19.6) & $32(30.8)$ & $36(41.4)$ & $79(32)$ & 0.024 \\
\hline Symptoms of an infection outside the CNS & $14(25)$ & $24(23.1)$ & $38(43.7)$ & $76(30.8)$ & 0.006 \\
\hline Headaches & $42(75)$ & $51(49)$ & $17(19.5)$ & $110(44.5)$ & $<0.001$ \\
\hline Neck stiffness & $19(33.9)$ & $31(29.8)$ & $31(35.6)$ & $81(32.8)$ & 0.68 \\
\hline Neck pain & $12(21.4)$ & $10(9.6)$ & $5(5.7)$ & $27(10.9)$ & 0.012 \\
\hline Vomiting & $20(35.7)$ & $20(19.2)$ & $10(11.5)$ & $50(20.2)$ & 0.002 \\
\hline Photophobia & $17(30.4)$ & $15(14.4)$ & $1(1.1)$ & $33(13.4)$ & $<0.001$ \\
\hline Phonophobia & $4(7.1)$ & $4(3.8)$ & $0(0)$ & $8(3.2)$ & - \\
\hline Focal neurological symptoms & $2(3.6)$ & $8(7.7)$ & $14(16.1)$ & $24(9.7)$ & 0.032 \\
\hline Altered consciousness & $2(3.6)$ & $18(17.3)$ & $12(13.8)$ & $32(13)$ & 0.046 \\
\hline Seizures & $3(5.4)$ & $21(20.2)$ & $5(5.7)$ & $29(11.7)$ & 0.003 \\
\hline \multicolumn{6}{|l|}{ Assumed primary LP indications } \\
\hline Infection of the CNS & $32(57.1)$ & $54(51.9)$ & $67(77)$ & $153(61.9)$ & 0.002 \\
\hline SAH & $18(32.1)$ & $33(31.7)$ & $12(13.8)$ & $63(25.5)$ & 0.008 \\
\hline Acute polyradiculoneuropathy (GBS) & $2(3.6)$ & $6(5.8)$ & $0(0)$ & $8(3.2)$ & - \\
\hline Undetermined & $4(7.1)$ & $11(10.6)$ & $8(9.2)$ & $23(9.3)$ & 0.78 \\
\hline $\mathrm{CT}$ prior to $\mathrm{LP}$ & $50(89.3)$ & $93(89.4)$ & $79(90.8)$ & $222(89.9)$ & 0.94 \\
\hline \multicolumn{6}{|l|}{ Diagnosis } \\
\hline Aseptic meningitis & $12(21.4)$ & $8(7.7)$ & $1(1.1)$ & $21(8.5)$ & - \\
\hline Bacterial meningitis & $1(1.8)$ & $3(2.9)$ & $2(2.3)$ & $6(2.4)$ & - \\
\hline Carcinomatous meningitis & $0(0)$ & $1(1)$ & $0(0)$ & $1(0.4)$ & - \\
\hline All meningitis & $13(23.2)$ & $12(11.5)$ & $3(3.4)$ & $28(11.3)$ & 0.002 \\
\hline SAH & $0(0)$ & $1(1)$ & $0(0)$ & $1(0.4)$ & - \\
\hline Acute polyradiculoneuropathy (GBS) & $1(1.8)$ & $2(1.9)$ & $1(1.1)$ & $4(1.6)$ & - \\
\hline All contributive LP & $14(25)$ & $15(14.4)$ & $4(4.6)$ & $33(13.4)$ & 0.003 \\
\hline
\end{tabular}

$L P$ lumbar puncture, $C N S$ central nervous system, $C T$ computed tomography of the brain, $p p$ value of the chi-square test (when applicable), $G B S$ acute Guillain-Barré syndrome

Neurological diseases, alcoholism, diabetes, and chronic headaches were the most frequent features in medical records (Table 1). Neurological diseases and diabetes were the most frequent features in elderly patients (aged above 65 years old). Alcoholism was overrepresented in the middle-aged patients (aged between 35 and 65 years old). Chronic headaches were the most frequent feature in the youngest patients (aged less than 35 years old).
Among patients' clinical features observed at admission or at the clinical examination, nonfocal neurological symptoms (delirium or acute confusion), fever, and symptoms conducive to an infection outside the CNS were the most frequent findings. Symptoms comprising the meningitis syndrome, such as headaches, neck stiffness or pain, vomiting, photophobia, and phonophobia, were also observed. These symptoms were overrepresented among young patients; headaches 
were reported by three quarters of young patients. Elderly patients more often presented with fever, clinical signs suggesting infections outside the CNS, and nonfocal neurological signs (mainly confusion).

Focal neurological signs, seizures, and abnormal consciousness were reported each for a little more than $10 \%$ of our population and were infrequently observed among the young patients. Middle-aged patients more frequently presented with seizures.

\section{LP indications}

The assumed indications for LP were principally the search for CNS infections $(61.9 \%$ of the cases) followed by the search for SAH (25.5\%) and GBS (3.2\%). The search for CNS infections were most frequent among the elderly patients, representing almost $80 \%$ of LP indications (Table 1). LP motivated by the search for SAH represented $32 \%$ of cases among patients of less than 65 years old versus $14 \%$ among older patients. LP indications were indeterminate or uncertain for a little less than $10 \%$ of our population.

Final diagnoses

Of the 247 patients who underwent an LP, CSF analysis confirmed 21 cases of aseptic (lymphocytic) meningitis (8.5\%), 6 cases of bacterial meningitis (2.4\%), and 1 case of SAH $(0.4 \%)$ (Table 1, Figs. 1 and 2). LP contributed to the diagnosis of GBS in four cases $(1.6 \%)$ and of carcinomatous meningitis in one case $(0.4 \%)$. Lymphocytic meningitis was more frequently observed among younger patients.

When the lumbar puncture results were not contributive (normal or negative), the final diagnoses were essentially infections outside the CNS (30\%), neurological diseases (28.7\%), and benign headaches (14.2\%).

The main sites reported suggestive of infections outside the CNS were pulmonary, urinary, and in the upper respiratory tracts. Pulmonary and urinary sites were more frequently observed among elderly patients, whilst upper respiratory tract sites were more often reported among young and middle-aged patients. Pulmonary $(34 \%)$ and urinary $(24 \%)$ infections represented the two main sites of infections outside the CNS. As expected, these infections were overrepresented among elderly patients, representing, respectively, 18 and $14 \%$ of final diagnoses.

Compared to the other patients, the youngest patients more frequently presented with benign viral infections $(7.1 \%$ of all diagnoses) and benign headaches (30.4\%). They also presented with fewer noninfectious neurological disorders: $16 \%$ of final diagnoses versus $38 \%$ among middle-aged patients and $30 \%$ among elderly patients $(p<0.05)$. Differential noninfectious neurological disorders were more often observed among older patients and were mostly seizures and strokes: they represented, among noninfectious neurological disorders, 44 and $24 \%$ of diagnoses, respectively. Seizures, representing a final diagnosis, were overrepresented among middle-aged patients (19\% of all diagnoses), whilst strokes were overrepresented among middle-aged and elderly patients ( $8 \%$ of all diagnoses).

\section{LP efficiency and CSF tests}

Contributive LP represented 13.4\% (95\% CI: 9.1-17.6) of the cases and varied significantly according to patients' age: the proportion of efficient LP decreased from $25 \%$ among young patients to $14.2 \%$ among middle-aged patients and to less than 5\% among elderly patients (Table 1 and Fig. 1).
Fig. 1 Final diagnoses defining efficient LP (black bars) and principal differential diagnoses (grey bars) in the presence of inefficient LP

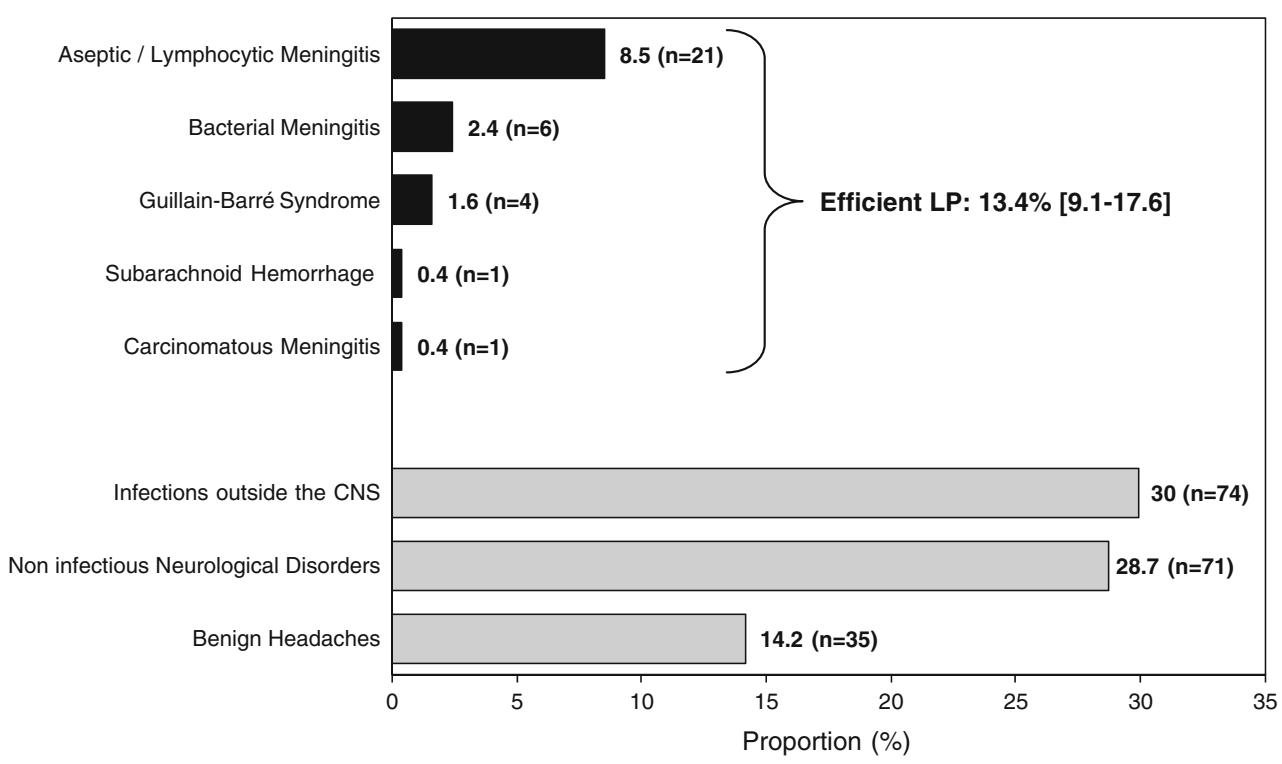


Fig. 2 Final distribution of main diagnoses according to age (proportion among each age category)

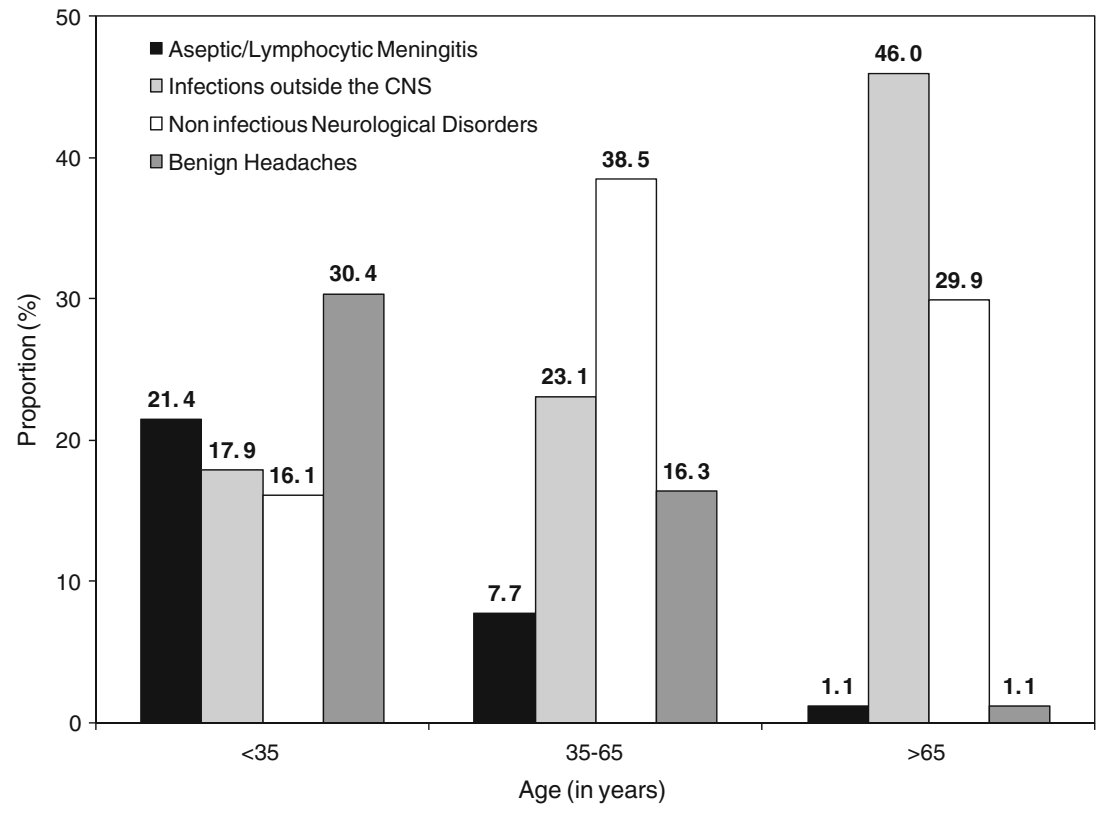

A large majority of these positive and contributive LP (63.6\%), confirming a suspected specific diagnosis in an EMD setting corresponded to a diagnosis of aseptic (lymphocytic) meningitis.

One of the cases of aseptic meningitis was concomitant with a clinical and serum diagnosis of an Epstein-Barr virus infection that was not confirmed by the CSF tests. The search for herpes simplex in CSF, using a herpes simplex polymerase chain reaction (PCR), was performed in 126 patients but was positive in only one case. Among the cases of bacterial meningitis $(n=6)$, three were caused by Streptococcus pneumoniae, one by Streptococcus agalactiae, and one by Staphylococcus aureus. Unfortunately, in one case, the bacteria could not be identified, whilst CSF characteristics were undoubtedly in accordance with a diagnosis of bacterial meningitis.

A comparison of standard CSF tests between aseptic (lymphocytic) and bacterial meningitis showed several consistent differences (Table 2).
Soluble antigens were detected in the CSF in all cases of pneumococcal meningitis, and the germ could also be identified immediately. This CSF test was ordered for only 15 patients.

Taking into account the cutoff points proposed by Shah et al. [22] for the red blood cell count in CSF, there were $10.1 \%(6.1-14.1)$ of traumatic LP when the cutoff value was $1,000 / \mathrm{mm}^{3}$ and $17 \%$ [12-22] when the cutoff value was $400 / \mathrm{mm}^{3}$.

Medical practices and complications related to LP use

Almost all patients underwent a CT prior to the LP $[89.9 \%$ (86.1-94.6)], independently of LP indications (Table 1). When LP were performed within the first $24 \mathrm{~h}$ after admission, the median delay between admission and cranial CT was $2 \mathrm{~h}$; the median delay for patients admitted between midnight and 6 A.M. was 7 h. The median delay between admission and LP results was $6 \mathrm{~h}$, whereas delays of up to $10 \mathrm{~h}$ were
Table 2 Comparison of biological test characteristics (medians) between bacterial and lymphocytic/aseptic meningitis ( $p$ value according to nonparametric tests)

\begin{tabular}{|c|c|c|c|}
\hline Test type/meningitis type & $\begin{array}{l}\text { Bacterial } \\
(n=6)\end{array}$ & $\begin{array}{l}\text { Lymphocytic } \\
(n=21)\end{array}$ & $p$ \\
\hline \multicolumn{4}{|l|}{ In blood serum } \\
\hline Leukocytes $(\mathrm{g} / \mathrm{l})$ & 17.2 & 10.1 & $<0.01$ \\
\hline $\mathrm{CRP}(\mathrm{mg} / \mathrm{l})$ & 139.5 & 8 & 0.01 \\
\hline \multicolumn{4}{|l|}{ In CSF } \\
\hline Leukocytes $/ \mathrm{mm}^{3}$ & 1,200 & 68 & $<0.01$ \\
\hline Blood cells $/ \mathrm{mm}^{3}$ & 100 & 64 & 0.23 \\
\hline Protein level (g/l) & 2.43 & 0.65 & $<0.01$ \\
\hline Glucose level (g/l) & 0.22 & 0.61 & $<0.001$ \\
\hline Ratio of glucose in CSF to glucose in blood serum & 0.15 & 0.58 & $<0.01$ \\
\hline Chloride level (mmol/l) & 116 & 123 & $<0.01$ \\
\hline
\end{tabular}


recorded for some of the patients admitted between midnight and 6 A.M. A positive correlation was found between the delay before cranial CT and the delay before obtaining LP results: the correlation coefficient was $0.6(p<0.0001)$.

In terms of LP complications, only postdural headaches were reported among patients admitted secondarily to the neurological and medical units. Postdural puncture headaches (PDPH) were reported in $6.1 \%(3.1-9.1)$ of patients who underwent an LP.

More than four fifths of patients were admitted to hospitalization units, whilst $14 \%$ were discharged from the EMD. When compared to patients admitted to hospitalization units, patients discharged from our EMD were much younger: $39 \%$ of discharged patients were less than 35 years old versus $20 \%$ among admitted patients. Discharged patients also presented more frequently with less severe final diagnoses such as benign headaches (36 versus $11 \%$ ) and mild psychiatric disorders (15 versus $3 \%$ ).

\section{Discussion}

Our study provides a relevant appraisal of the use and the diagnostic efficiency of LP in an EMD setting. LP was performed occasionally in $0.5 \%$ of the patients admitted to our adult EMD. The assessment of final diagnoses indicates a modest efficiency (yield) of LP, namely in fewer than $15 \%$ of cases. Among efficient LP, aseptic (lymphocytic) meningitis was the most frequent diagnosis, whereas the search for an infection of the CNS was the most frequent indication for LP. Thus, patients could benefit from optimal treatments and strategies of care, based upon the results of CSF analyses. In cases of ineffective LP, the principal differential diagnoses were infections outside the CNS, noninfectious neurological disorders (seizures and strokes), and benign headaches.

Though LP efficiency decreased dramatically according to age, differential diagnoses (diagnoses found in cases of inefficient LP) also varied substantially according to patients' age. Differential diagnoses among elderly patients were principally represented by infections outside the CNS, mainly in the urinary and respiratory tracts, and by seizures and strokes. Middle-aged patients presented more frequently with seizures and benign headaches as differential diagnoses. Benign headaches represented the most frequent final diagnosis among young patients. Observed infectious diseases outside the CNS were located mostly in the upper respiratory tracts among middle-aged and young patients.

Considering the seriousness of meningitis, $\mathrm{SAH}$, and acute GBS disorders, which may compromise patients' functional and vital prognoses, LP represent a consistent tool to rule out SAH [16] or meningitis [3] and to support a diagnosis of suspected acute GBS [23] in an EMD setting.
Therefore, despite the modest efficiency of CSF examinations revealed by our results, negative or normal CSF findings can be informative and useful. However, before performing an LP, the main differential diagnoses should be considered, so as to improve the justification and indication for the LP procedure. This is particularly important for the elderly patients whose LP yield is very weak ( $5 \%$ of cases). Nevertheless, the modest specific diagnostic efficiency of LP observed in our study (13.4\%) remains difficult to interpret. It can represent a balance between consistent resource utilization versus misdiagnosis.

Our results were in line with the findings of the few published studies exploring LP use and efficiency in EMD settings. The frequency of LP use found in our study was comparable to that found by Mòdol et al. $(0.4 \%)$ [8]. The distribution of efficient LP diagnoses in our study was also in line with the results of Mòdol et al.'s study [8], which found that the principal indication for LP in an adult EMD was a suspected CNS infection (76\%). Only $30 \%$ of the CSF examinations confirmed the diagnosis of acute meningitis among LP performed to search for a CNS infection. A modest efficiency of LP has also been reported by Powers [1] and Warshaw et al. [10]. Warshaw et al.'s study found a very weak efficiency of CSF analysis among elderly patients presenting with fever and delirium; the principal final diagnoses were pulmonary, urinary, and viral infections, dehydration, and metabolic causes. After an appraisal of 104 CSF examinations, Powers [1] also found a modest efficiency of LP performed in an EMD, almost all CSF tests giving normal or negative results. Moreover, the author of that study recommended limiting the laboratory testing of CSF.

A low incidence of GBS in an emergency setting was recently reported by McGillicuddy et al. [5]. In addition, an acute GBS diagnosis is usually confirmed by electrodiagnostic testing [23]. As is the case for GBS, SAH [9] diagnoses using LP results are infrequent in the EMD. Occurrences of GBS and SAH observed in our study are supported by Perry et al.'s [9] and McGillicuddy et al.'s [5] findings. Nonetheless, difficulties in diagnosing these pathological conditions have been reported [4, 5, 11, 24].

The commonest complication of LP is PDPH [25], whereas other, more serious complications have exceptionally been observed, such as cerebral herniation [26], spinal hematoma [27], iatrogenic spinal tumor [28], and induced meningitis [29]. The incidence of PDPH after a diagnostic LP procedure can vary from 6 to $40 \%[30,31]$. In our survey, a rate of PDPH of $6.1 \%$ was found. No other LP complications have been observed. However, our rate of PDPH may be underestimated. Information not reported in patients' files and early discharge from the EMD to home (14\% of patients) represent potential mechanisms of misclassification. 
Significant differences between aseptic and bacterial meningitis were found in our study, when standard CSF tests were considered. While standard CSF tests are known to provide valuable support in differentiating bacterial from aseptic (lymphocytic) meningitis [32], some authors have suggested that these standard tests are only moderately useful in the event of a negative Gram-stained smear [19, 33, 34]. Other specific CSF and blood serum tests have been proposed to better appreciate the likelihood of bacterial meningitis $[18,35]$.

The usefulness of the occurrence of soluble antigens in CSF was limited in our survey; however, this CSF test was infrequently employed ( $6 \%$ of CSF) in accordance with published data [36-39]. Very low efficiency was achieved with the use of herpes simplex PCR in our study. This result suggests an excessive use of this CSF test $(51 \%$ of CSF, one positive result). Excessive herpes simplex PCR testing is linked to the frequent use of this test in patients whose CSF did have a normal count of leukocytes (in 81 of 126 cases). Ordering this test may have been systematic to rule out a very weak likelihood of herpes simplex meningoencephalitis [20].

Taking into account the blood cell count cutoff points proposed by Shah et al. [22], the rates of traumatic LP observed in our study were similar to previously published data.

Among elderly patients, fewer than 5\% of LP were efficient, whilst almost four fifths of LP were employed to search for a CNS infection. The majority of infections detected among elderly patients were principally located in the respiratory or urinary tracts. Moreover, clinical signs notably suggesting infections located outside the CNS were found in fewer than half $(41 \%)$ of the elderly patients.

Greater yield of LP was found among young $(25 \%$ of cases) and middle-aged patients (a little less than $15 \%$ of cases). Among these subpopulations, efficient LP documented primarily infections of the CNS (mainly aseptic meningitis). Younger patients more frequently presented with obvious clinical signs of meningism. LP indications among these patients may have corresponded to a higher clinical suspicion of meningitis. In contrast to the younger patients, elderly patients may have undergone LP to rule out the possibility of a CNS infection. Clinical signs suggesting a diagnosis of meningitis or encephalitis can be misleading in elderly patients [40]. Clinical suspicion of CNS infection is assumed to have differed widely between these subpopulations and can explain the differences observed in LP efficiency. In addition, when LP were performed to rule out an assumed weak likelihood of meningitis, markedly among elderly patients, brain $\mathrm{CT}$ and LP may have been delayed.

The usefulness of performing a CT prior to an LP depends on the patient's clinical condition and the suspected diagnosis. Fitch et al. [3] reported on patients' conditions that require a CT to be performed before an LP when a CNS infection is suspected, whereas Hasbun et al. [15] showed that patients' clinical features can reliably be used to assess the usefulness of CT. Furthermore, a very low prevalence of abnormal $\mathrm{CT}$, contraindicating LP, was observed in a study of 113 patients admitted to an EMD who were considered to require an LP [41]. Nonetheless, many clinicians routinely order a CT prior to LP [14, 42]. Although this strategy may be reassuring, it may sometimes lead to a substantial delay in diagnosis and thus in optimal treatment, especially when the availability of CT is limited $[9,43]$. In addition, this practice may lead to clinical contraindications being overlooked [14].

With regard to medical practices related to the use of LP, in our study, a brain CT prior to LP seems to be routinely employed: clinical signs (focal neurological signs, seizures, or abnormal consciousness) requiring a brain $\mathrm{CT}$ prior to the performance of an LP were found among less than a third of our population. Unexpectedly, approximately $90 \%$ of patients who underwent an LP to search for an infection of the CNS had CT prior to LP. Significant delays have been observed between admission, the brain CT, and LP results, especially when admissions occurred between midnight and 6 A.M. Flais et al. [43] reported a huge decrease in $\mathrm{CT}$ use between midnight and 6 A.M. for emergency demands. When LP are performed in order to search for an infection of the CNS, ordering a CT prior to an LP can lead to an important delay of the LP procedure. Thus, administration of antibiotics may also be delayed, compromising the prognosis of patients with bacterial meningitis [44].

In the event of a negative brain $\mathrm{CT}$, it is recommended to wait for at least $6 \mathrm{~h}$ between the onset of symptoms and the performance of an LP when SAH is suspected [6]. This practice is in accordance with observed CT and LP procedure delays in our survey. SAH diagnoses are more frequently confirmed using brain imaging [9], which is usually performed as the first-line diagnostic tool [45]. However, in spite of brain CT enhancement using fifth generation CT scanners, which improves the diagnosis of SAH [46], the sensitivity of noncontrast CT imaging may, in some cases, remain insufficient [13]. Hence, the use of LP remains necessary to rule out SAH [16]. However, LP are not reported to be systematically used in the event of negative brain CT $[4,9]$. Furthermore, whether an LP is indicated in the event of a negative brain CT when a SAH is suspected has been recently disputed $[6,47,48]$. Vermeulen et al. [11] assessed the rate of missed SAH among emergency department patients; a rate of 5.4\% (4.3-6.6) was found among a population of 1,507 patients.

In our study, assumed SAH indications for LP represented only $25.5 \%$ of the cases and only one case of SAH 
was found using an LP. SAH indications were more frequent among middle-aged and young patients. Headaches, as a record or a symptom observed during the clinical examination, were overrepresented among these patients. Although missed SAH diagnosis could not be studied in our survey, very infrequent diagnosis of SAH from LP results can be linked to the effectiveness of brain imaging diagnosis (when CT are performed prior to LP) and limited indications for LP in relation to emergency physicians' strategy of care.

Several limitations, linked to the retrospective nature of our study, must be considered when assessing our results. LP subsequently performed in hospitalization units after an initial admission in the EMD were not taken into account, leading to a potential selection bias. However, LP performance in the hospitalization units could be influenced by changes in clinical or biological features. In this study, our aim was to assess emergency medical practices in relation to patients' initial characteristics observed in the EMD. Classification and information biases can result from the retrospective reviewing of patients' medical files, leading to a more difficult assessment of patients' features, indications for LP, and final diagnoses. Thus, two emergency physicians reviewed patients' medical files independently and the concordance of our results with the literature data suggests that these biases are limited.

A better assessment of the efficiency of LP would undoubtedly be achieved in a prospective setting with a larger study population. This would allow a more precise and reliable assessment of LP indications, patients' features, delivery of anti-infective treatments (in cases of suspected meningitis), and outcomes during a follow-up. Missed meningitis and SAH diagnoses, in the EMD, would also be appreciated. The assessment of medical practices, illustrated by the use of brain CT prior to LP (in cases of a possible infection of the CNS) and herpes simplex PCR tests, suggests that the current published guidelines and data were not fully taken into account. A prospective survey would permit evaluation of potential improvements in medical practices after a review of current guidelines.

Acknowledgments The authors would like to thank Nick Barton, Manuella Nuytens, Laureen Ribassin-Majed and Elvira Escrich for their invaluable help.

Conflicts of interest None.

\section{References}

1. Powers RD (1987) Emergency department lumbar puncture: clinical application and utility of laboratory testing. Am J Emerg Med 5(6):516-520

2. Sharief M (2004) Lumbar puncture and CSF examination. Medicine 32(9):44-46
3. Fitch MT, van de Beek D (2007) Emergency diagnosis and treatment of adult meningitis. Lancet Infect Dis 7(3):191-200

4. O’Neill J, McLaggan S, Gibson R (2005) Acute headache and subarachnoid haemorrhage: a retrospective review of $\mathrm{CT}$ and lumbar puncture findings. Scott Med J 50(4):151-153

5. McGillicuddy DC, Walker O, Shapiro NI, Edlow JA (2006) Guillain-Barré syndrome in the emergency department. Ann Emerg Med 47(4):390-393

6. Al-Shahi R, White PM, Davenport RJ, Lindsay KW (2006) Subarachnoid haemorrhage. BMJ 333(7561):235-240

7. Fletcher DD, Lawn ND, Wolter TD, Wijdicks EF (2000) Longterm outcome in patients with Guillain-Barré syndrome requiring mechanical ventilation. Neurology 54(12):2311-2315

8. Mòdol JM, Tudela P, Veny A, Sahuquillo JC, Tor J, Giménez M (2002) Indications and diagnosis efficiency of lumbar puncture in emergencies (in Spanish). Med Clin (Barc) 118(1):10-12

9. Perry JJ, Stiell I, Wells G, Spacek A (2002) Diagnostic test utilization in the emergency department for alert headache patients with possible subarachnoid hemorrhage. CJEM 4(5):333-337

10. Warshaw G, Tanzer F (1993) The effectiveness of lumbar puncture in the evaluation of delirium and fever in the hospitalized elderly. Arch Fam Med 2(3):293-297

11. Vermeulen MJ, Schull MJ (2007) Missed diagnosis of subarachnoid hemorrhage in the emergency department. Stroke 38(4):1216-1221

12. Baker ND, Kharazi H, Laurent L, Walker AT, Williamson DS, Weissman BN, Zamani A, Sanchez R (1994) The efficacy of routine head computed tomography (CT scan) prior to lumbar puncture in the emergency department. J Emerg Med 12(5):597-601

13. Byyny RL, Mower WR, Shum N, Gabayan GZ, Fang S, Baraff LJ (2008) Sensitivity of noncontrast cranial computed tomography for the emergency department diagnosis of subarachnoid hemorrhage. Ann Emerg Med 51(6):697-703

14. Cooper JR (1999) Routine use of CT prior to lumbar puncture. $\mathrm{Br}$ J Radiol 72(855):319

15. Hasbun R, Abrahams J, Jekel J, Quagliarello VJ (2001) Computed tomography of the head before lumbar puncture in adults with suspected meningitis. N Engl J Med 345(24):1727-1733

16. Perry JJ, Spacek A, Forbes M, Wells GA, Mortensen M, Symington C, Fortin N, Stiell IG (2008) Is the combination of negative computed tomography result and negative lumbar puncture result sufficient to rule out subarachnoid hemorrhage? Ann Emerg Med 51(6):707-713

17. van Crevel H, Hijdra A, de Gans J (2002) Lumbar puncture and the risk of herniation: when should we first perform CT? J Neurol 249(2):129-137

18. Berg B, Gärdsell P, Skånsberg P (1982) Cerebrospinal fluid lactate in the diagnosis of meningitis. Diagnostic value compared to standard biochemical methods. Scand J Infect Dis 14(2):111-115

19. Ray P, Badarou-Acossi G, Viallon A, Boutoille D, Arthaud M, Trystram D, Riou B (2007) Accuracy of the cerebrospinal fluid results to differentiate bacterial from non bacterial meningitis, in case of negative gram-stained smear. Am J Emerg Med 25(2):179-184

20. Tyler KL (2004) Herpes simplex virus infections of the central nervous system: encephalitis and meningitis, including Mollaret's. Herpes 11(Suppl 2):57A-64A

21. Vittinghoff E (2005) Regression methods in biostatistics: linear, logistic, survival, and repeated measures models. Springer, New York

22. Shah KH, Richard KM, Nicholas S, Edlow JA (2003) Incidence of traumatic lumbar puncture. Acad Emerg Med 10(2):151-154

23. Burns TM (2008) Guillain-Barré syndrome. Semin Neurol 28 (2):152-167

24. Perry JJ, Stiell IG, Wells GA, Mortensen M, Lesiuk H, Sivilotti M, Kapur A (2005) Attitudes and judgment of emergency physicians in the management of patients with acute headache. Acad Emerg Med $12(1): 33-37$ 
25. Sternbach G (1985) Lumbar puncture. J Emerg Med 2(3):199-203

26. Oliver WJ, Shope TC, Kuhns LR (2003) Fatal lumbar puncture: fact versus fiction - an approach to a clinical dilemma. Pediatrics 112(3 Pt 1):e174-e176

27. Peltola J, Sumelahti ML, Kumpulainen T, Dastidar P, Helén P (1996) Spinal epidural haematoma complicating diagnostic lumbar puncture. Lancet 347(8994):131

28. Park JC, Chung CK, Kim HJ (2003) Iatrogenic spinal epidermoid tumor. A complication of spinal puncture in an adult. Clin Neurol Neurosurg 105(4):281-285

29. Pandian JD, Sarada C, Radhakrishnan VV, Kishore A (2004) Iatrogenic meningitis after lumbar puncture - a preventable health hazard. J Hosp Infect 56(2):119-124

30. Seupaul RA, Somerville GG, Viscusi C, Shepard AJ, Hauter WE (2005) Prevalence of postdural puncture headache after ED performed lumbar puncture. Am J Emerg Med 23(7):913-915

31. Frank RL (2008) Lumbar puncture and post-dural puncture headaches: implications for the emergency physician. J Emerg Med 35:149-157

32. Hoen B, Viel JF, Paquot C, Gérard A, Canton P (1995) Multivariate approach to differential diagnosis of acute meningitis. Eur J Clin Microbiol Infect Dis 14(4):267-274

33. Lindquist L, Linné T, Hansson LO, Kalin M, Axelsson G (1988) Value of cerebrospinal fluid analysis in the differential diagnosis of meningitis: a study in 710 patients with suspected central nervous system infection. Eur J Clin Microbiol Infect Dis 7(3):374-380

34. Spanos A, Harrell FE Jr, Durack DT (1989) Differential diagnosis of acute meningitis. An analysis of the predictive value of initial observations. JAMA 262(19):2700-2707

35. Dubos F, Moulin F, Gajdos V, De Suremain N, Biscardi S, Lebon P, Raymond J, Breart G, Gendrel D, Chalumeau M (2006) Serum procalcitonin and other biologic markers to distinguish between bacterial and aseptic meningitis. J Pediatr 149(1):72-76

36. Camargos PA, Almeida MS, Filho GL, Batista KW, Carvalho AG, Pereira CL (1994) Blood stained cerebrospinal fluid responsible for false positive reactions of latex particle agglutination tests. J Clin Pathol 47(12):1116-1117

37. Sippel JE, Hider PA, Controni G, Eisenach KD, Hill HR, Rytel MW, Wasilauskas BL (1984) Use of the directigen latex agglutination test for detection of Haemophilus influenzae, Streptococcus pneumoniae, and Neisseria meningitidis antigens in cerebrospinal fluid from meningitis patients. J Clin Microbiol 20(5):884-886

38. Coovadia YM, Naidu KK (1985) Evaluation of Bactigen latex agglutination and Phadebact coagglutination for detection of bacterial antigens in cerebrospinal fluid. J Clin Pathol 38(5):561-564

39. Coovadia YM, Solwa Z (1987) Three latex agglutination tests compared with gram staining for the detection of bacteria in cerebrospinal fluid. S Afr Med J 71(7):442-444
40. Shah K, Richard K, Edlow JA (2007) Utility of lumbar puncture in the afebrile vs. febrile elderly patient with altered mental status: a pilot study. J Emerg Med 32(1):15-18

41. Gopal AK, Whitehouse JD, Simel DL, Corey GR (1999) Cranial computed tomography before lumbar puncture: a prospective clinical evaluation. Arch Intern Med 159(22):2681-2685

42. Clark T, Duffell E, Stuart JM, Heyderman RS (2006) Lumbar puncture in the management of adults with suspected bacterial meningitis - a survey of practice. J Infect 52(5):315-319

43. Flais S, Lasar Y, Carré P, Bordet F, Huynh TL (1999) Emergency computed tomography in a general hospital center (in French). J Radiol 80(5):441-446

44. Aronin SI, Peduzzi P, Quagliarello VJ (1998) Communityacquired bacterial meningitis: risk stratification for adverse clinical outcome and effect of antibiotic timing. Ann Intern Med 129(11):862-869

45. Pancu D, Davenport M, Roth K, Heller M (2004) EPs do not accept the strategy of "lumbar puncture first" in subarachnoid hemorrhage. Am J Emerg Med 22(2):115-117

46. Boesiger BM, Shiber JR (2005) Subarachnoid hemorrhage diagnosis by computed tomography and lumbar puncture: are fifth generation CT scanners better at identifying subarachnoid hemorrhage? J Emerg Med 29(1):23-27

47. Coats TJ, Loffhagen R (2006) Diagnosis of subarachnoid haemorrhage following a negative computed tomography for acute headache: a Bayesian analysis. Eur J Emerg Med 13(2):80-83

48. Al-Shahi R, White PM, Davenport RJ, Lindsay KW (2006) Subarachnoid haemorrhage: lumbar puncture for every negative scan? Authors' reply. BMJ 333(7567):550

Bilal Majed is a consultant Medical Doctor (MD) at the Emergency Department of the Arras Hospital in France. He is experienced as an emergency medicine practitioner since five years and takes part to clinical activities in the Emergency room, the Intensive Care Unit, the Pre-hospital Mobile Field Care Unit and in the patient dispatching center for emergency calls that manage pre-hospital emergencies. Bilal Majed is also in charge of the recently created (2009) Epidemiology and Clinical Research Unit of the Emergency Department of the Arras Hospital. He graduated as a MD from the University of Lille II (Lille, France) and as an Emergency Medicine specialist; he is also specialized in Epidemiology $(\mathrm{PhD})$ from Paris XI University (Paris, France). Though he explored previously an epidemiological issue in the field of breast cancer (prognosis value of obesity in breast cancer), he extended his actual research activities to studies in the field of emergency medicine (observational epidemiologic studies, clinical trials and assessment of medical practices). 